\title{
Editorial
}

\section{Treat-to-Target in Systemic Lupus Erythematosus: The Time Is Now!}

\author{
M. Salah Eldin Abdel Baky \\ Department of Internal Medicine, Division of Rheumatology, Ain Shams University; Egypt
}

Until the 1950s, 5-year survival of systemic lupus erythematosus (SLE) patients was around 50\%, making patient survival the primary (if not the only) target for treatment. Over the decades, a sharp increase of patient survival has been observed, leading to a change in disease characteristics and the emergence of a number of issues that may affect patient outcomes, such as damage accrual, comorbidities management, and patient's quality of life. Despite significant advances in our understanding of the pathogenesis and clinical course of systemic lupus erythematosus (SLE), and a notable improvement in overall survival, the prospects for patients with this disease remain disappointing. ${ }^{1}$

Chronic and persistent symptoms, the pervasive risk for flares, the need for such long-term therapies as corticosteroids, and disease-related end-organ damage all contribute to significant reductions in health-related quality-of-life (HR-QOL) in SLE.

The fact that a high percentage of patients have persistently active disease may suggest one of two explanations: that the available drugs are not able to completely control disease activity, or that physicians are keen to accept a compromise between an acceptable level of disease activity and the risk of additional toxicity and probably damage induced by available drugs. ${ }^{2}$

In routine clinical practice, the treating physician based on her /his experience assesses disease activity. Validated indices have been developed to measure the degree of activity of SLE and these indices are generally used in research and randomized controlled trials. Some recommendations suggest their use also in routine clinical practice. Cut-off values have been proposed to classify disease activity and define response to therapy based on these indices. However, no agreement has been reached yet concerning specific levels as predetermined targets for treatment or a definition of remission ${ }^{3}$.

Patients with rheumatic diseases, including SLE, are at increased risk of comorbidities, such as cardiovascular disease (CAD), infections, cancer, osteoporosis, which affect prognosis and survival; however, these conditions appear often underdiagnosed in routine clinical practice.

Damage, being due to active inflammation as well as to drugs or comorbidities, is an important prognostic factor for death and has a clear impact on the burden of disease for patients. ${ }^{4,5}$

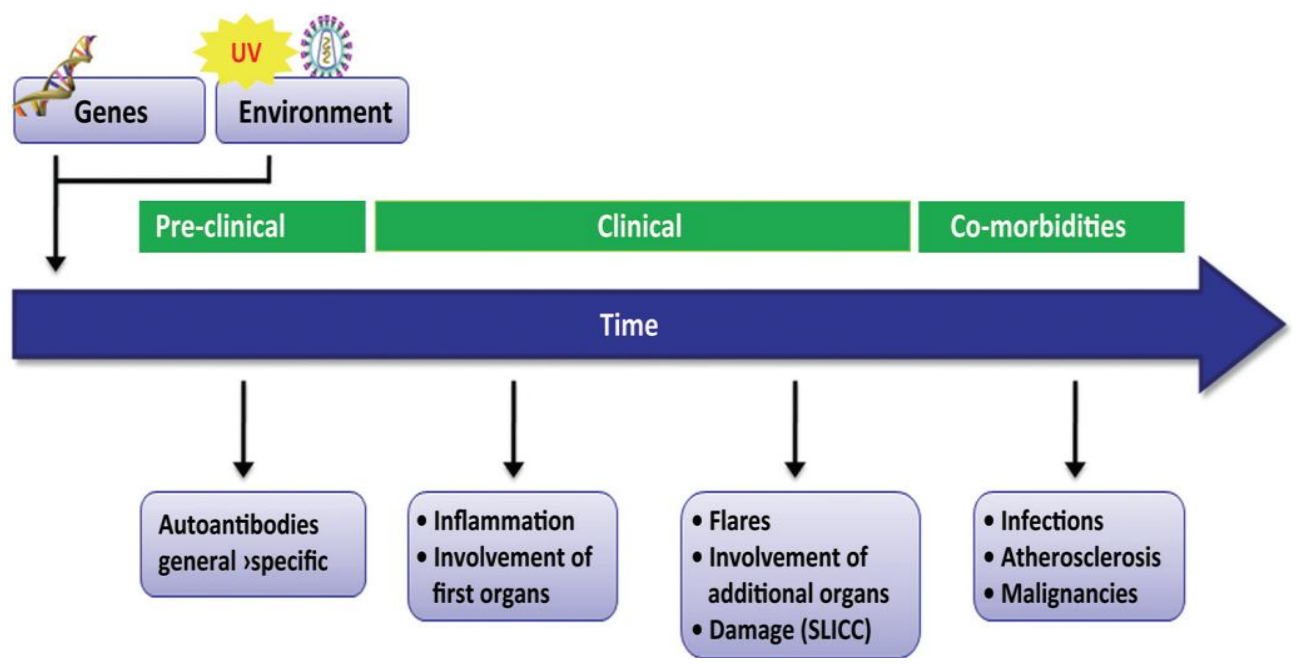

Natural history of systemic lupus erythematosus. Ann Rheum Dis 2010; 69:1603-11.

For Correspondence: e-mail: Salahym50@Yahoo.com 
These observations raise the question of how to achieve better outcomes of therapy. One reply could be newer and more effective therapeutic agents, and this is undoubtedly true. ${ }^{6}$ However, another important possibility is that currently available therapeutics could be used more effectively. This possibility is suggested by data from other therapeutic areas, where treating to target has yielded results superior to usual care.

\section{The principle of treating-to-target}

Treating to target is defining a therapeutic goal (the target), measuring it, and adjusting therapy based on whether the goal has been achieved. Treating to target is very similar to the idea of "tight control."

The treat-to-target concept originated in fields of medicine outside of rheumatology. It has been understood for decades that in treating hypertension, the purpose should not be to relieve symptoms, but to achieve risk reduction by meeting a specific blood pressure target. ${ }^{7}$ Similarly, in diabetes, targeting a specific A1C value (an easily measured goal), and using any of a large number of anti-diabetes medications to achieve the target, have improved outcomes. $^{8}$

In the field of rheumatology, In rheumatoid arthritis (RA), multiple clinical trials performed over 20 years have demonstrated clearly that patients have better outcomes if their disease activity at each timepoint for follow up is analyzed according to a prespecified target (usually remission or low disease activity, defined quantitatively through a disease activity index), adjusting therapy if the target has not been met. ${ }^{9}$

After the demonstration of the superiority of the strategy, an international expert panel published treatto-target recommendations for RA in $2010 .{ }^{10}$ These recommendations have been extensively discussed and are increasingly being implemented in the care of patients with this disease.

Since then, treat-to-target recommendations have also been developed for psoriatic arthritis and spondyloarthritis. ${ }^{11}$ These developments raised the question of whether treating to target would be applicable to the care of patients with SLE as well, and this was the starting point for the international expert panel in 2012.

The recommendations are solidly based on data from the literature obtained through systematic investigations of a list of key topics. The breadth and strength of the scientific data underlying each recommendation varied: For some, more than 100 citations were reviewed, whereas others were supported by only a few citations. Nevertheless, the scientific strength of the recommendations was generally very good, as was the degree of agreement with each recommendation among the expert/patient panel.
The Treat-to-target in systemic lupus erythematosus recommendations, which published in $2014^{12}$, clearly identify several possible targets that could be used in a treat-to-target approach for SLE. Remission should be targeted if possible, but prevention of flares, prevention or minimization of damage, reduction of corticosteroid use, and improvements in overall HR-QOL are all identified as reasonable therapy targets. The decision of which target to select at which point in time for each patient will remain the key challenge for the physician taking care of patients with SLE.

The recommendations also provide specific pointers to therapy in general, specifying the important role of immunosuppressive agents in the management of lupus nephritis, the general role of antimalarial agents, the need to address comorbid conditions, and the importance of managing concomitant antiphospholipid syndrome.

\section{Treat-to-target in systemic lupus erythematosus: overarching principles and bullet points ${ }^{12}$}

\section{Overarching principle}

1. The management of systemic lupus erythematosus (SLE) should be based on shared decisions between the informed patient and her/his physician(s).

2. Treatment of SLE should aim at ensuring longterm survival, preventing organ damage, and optimizing health-related quality-of-life, by controlling disease activity and minimizing comorbidities and drug toxicity.

3. The management of SLE requires an understanding of its many aspects and manifestations, which may have to be targeted in a multidisciplinary manner.

4. Patients with SLE need regular long-term monitoring and review and/or adjustment of therapy.

\section{Recommendations}

1. The treatment target of SLE should be remission of systemic symptoms and organ manifestations or, where remission cannot be reached, the lowest possible disease activity, measured by a validated lupus activity index and/or by organspecific markers.

2. Prevention of flares (especially severe flares) is a realistic target in SLE and should be a therapeutic goal.

3. It is not recommended that the treatment in clinically asymptomatic patients be escalated based solely on stable or persistent serological activity. 
4. Since damage predicts subsequent damage and death, prevention of damage accrual should be a major therapeutic goal in SLE.

5. Factors negatively influencing health-related quality of life (HRQOL), such as fatigue, pain and depression should be addressed, in addition to control of disease activity and prevention of damage.

6. Early recognition and treatment of renal involvement in lupus patients is strongly recommended.

7. For lupus nephritis, following induction therapy, at least 3 years of immunosuppressive maintenance treatment is recommended to optimize outcomes.

8. Lupus maintenance treatment should aim for the lowest glucocorticoid dosage needed to control disease, and if possible, glucocorticoids should be withdrawn completely.

9. Prevention and treatment of antiphospholipid syndrome (APS)-related morbidity should be a therapeutic goal in SLE; therapeutic recommendations do not differ from those in primary APS.

10. Irrespective of the use of other treatments, serious consideration should be given to the use of antimalarials.

11. Relevant therapies adjunctive to any immunomodulation should be considered to control comorbidity in SLE patients.

\section{Where are we today?}

An important criticism of applying the treat-totarget concept in SLE is the fact that SLE is far more complex than hypertension. Therefore, it might be better to compare treating to target in SLE with treating to target in the entire field of cardiovascular disease prevention, where this strategy can be applied to multiple risk factors including hypertension, hypercholesterolemia, smoking and obesity. In the T2T/SLE recommendations, it is implied that several targets could be selected for each patient, each with its own "target value," timeline, and intervention. This is more similar to a treat-to-target approach for overall cardiovascular disease prevention than that for hypertension alone. $^{13}$

Paradoxically, even though the T2T/SLE task force strongly supported the use of remission as a conceptual target in SLE, there is currently no widely accepted definition of remission in SLE. An international task force recently started a long-term project to achieve consensus and a proposed definition for remission in SLE. Meanwhile, the Asia-Pacific Lupus Collaboration has developed a Lupus Low Disease Activity State (LLDAS) score. ${ }^{13}$

As new therapeutic options are becoming available, to which extent low disease activity would still be "acceptable", and if so, which "level" of activity for which period would be acceptable. ${ }^{14}$

A further critical issue is whether treating to target does in fact result in better outcomes in SLE. This has not yet been proved; the equivalent trial to TICORA for RA has not been performed for SLE. There have been serious discussions on the possibility of performing a randomized clinical trial comparing standard care and treat-to-target care.

In the process of developing T2T/SLE recommendations, experts have recognized that the key elements of treating to target are all possible to a lesser degree in SLE than in RA or in other diseases in which this approach has been applied successfully. Nevertheless, the introduction of treat-to-target principles to the care of patients with SLE cannot be postponed. Even if the work so far represents only a small portion of what still has to be done, it is undoubtedly the first important step toward a better treatment paradigm for SLE. ${ }^{13}$

\section{REFERENCES}

1. MAK A, CHEUNG MWL, CHIEW HJ, LIU YL, CHUN.MAN HO R: Global trend of survival in addition, damage of systemic lupus erythematosus: meta-analysis and meta regression of observational studies from the 1950s to 2000s. Semin Arthritis Rheum 2012; 41: 830- 49.

2. NOSSENT J, KISS E, ROZMAN B et al.: Disease activity and damage accrual during the early disease course in a multinational inception cohort of patients with systemic lupus erythematosus. Lupus 2010; 19: 949-56

3. American College of Rheumatology AD HOC Committee on Systemic Lupus Erythematosus Response Criteria: The American College of Rheumatology response criteria for systemic lupus erythematosus clinical trials: measures of overall disease activity. Arthritis Rheum 2004; 50: 3418-26.

4. MOSCA M, TANI C, ARINGER $M$ et al.: European League Against Rheumatism recommendations for monitoring patients with systemic lupus erythematosus in clinical practice and in observational studies. Ann Rheum Dis 2010; 69: 1269-74.

5. BERTSIAS G, IOANNIDIS JPA, BOLETIS $\mathrm{J}$ et al.: EULAR recommendations for the management of systemic lupus erythematosus. Ann Rheum Dis 2008; 67: 195-205.

6. van Vollenhoven RF, Parodis I, and Levitsky A. Biologics in SLE: towards new approaches. Best Pract Res Clin Rheumatol. 2013; 27:341-349.

7. Verdecchia P, Staessen JA, Angeli F, et al; CardioSis investigators. Usual versus tight control of systolic blood pressure in non-diabetic patients with hypertension (Cardio-Sis): an open-label randomized trial. Lancet. 2009; 374:525-533. 
8. Nathan DM, Cleary PA, Backlund JY, et al; Diabetes Control and Complications Trial/Epidemiology of Diabetes Interventions and Complications (DCCT/EDIC) Study Research Group. Intensive diabetes treatment and cardiovascular disease in patients with type 1 diabetes. N Engl J Med. 2005; 353:2643-2653.

9. Grigor C, Capell H, Stirling A et al.: Effect of a treatment strategy of tight control for rheumatoid arthritis (the TICORA study): a single blind randomized controlled trial. Lancet 2004; 364: 263 269.

10. Smolen JS, Aletaha D, Bijlsma JW, et al; T2T Expert Committee. Treating rheumatoid arthritis to target: recommendations of an international task force. Ann Rheum Dis. 2010;69:631-637
11. Smolen JS, Braun J, Dougados M, et al. Treating spondyloarthritis, including ankylosing spondylitis and psoriatic arthritis, to target: recommendations of an international task force. Ann Rheum Dis. 2014; 73:6-16.

12. Van Vollenhoven RF, Mosca M, Bertsias G, et al. Treat-to-target in systemic lupus erythematosus: recommendations from an international task force. Ann Rheum Dis. 2014; 73:958-967.

13. Ronald F. van Vollenhoven: An Important Shift in Lupus Care, Medscape.com July 21, 2014.

14. Mosca M, Boumpas DT, and Bruce IN, et al. Treatto-target in systemic lupus erythematosus: where are we today? Clin Exp Rheumatol. 2012; 30(4 Suppl 73): S112-S115. 